\title{
Theoretical study of single molecule fluorescence in a metallic nanocavity
}

\author{
Jörg Enderlein ${ }^{\text {a) }}$ \\ Institute for Biological Information Processing 1, Forschungszentrum Jülich, D-52425, Jülich, Germany
}

(Received 7 September 2001; accepted for publication 20 November 2001)

\begin{abstract}
A theoretical study is presented on the fluorescence excitation and emission of a molecule embedded within a nanometric metal-coated dielectric bead. It occurs that, for favorable values of bead radius and metal layer thickness, photostability of the molecule is improved significantly, due to a dramatic decrease of the fluorescence lifetime caused by the strong electromagnetic interaction between the emitting molecule and the metal layer. Moreover, the metal shell enhances the exciting electric field intensity leading to an increase in fluorescence brightness by more than one order of magnitude.

(c) 2002 American Institute of Physics. [DOI: 10.1063/1.1434314]
\end{abstract}

It is well known that the fluorescence emission properties of molecules can be dramatically changed by the presence of metals within wavelength distance (near field region). ${ }^{1,2}$ Due to the electromagnetic interaction of the emitting dye with the metal, the spontaneous emission rate can be enhanced or inhibited, depending on the exact dye-metal geometry. ${ }^{3}$ This change of excited state lifetime also affects the photostability of the dye. If one assumes that photobleaching of a dye takes place only while it is in the optically excited state, an enhanced emission rate (shorter excited state lifetime) results in an enhanced photostability. ${ }^{4}$ However, a significant part of the electromagnetic energy emitted by the dye is absorbed within the metal, and is thus lost for fluorescence detection. Nonetheless, as was shown in detail for planar metal/dielectric systems, under favorable geometrical conditions, higher photostability without severely compromising the detectable fluorescence intensity can be achieved, particularly if the metal is also exploited for enhancing the local intensity of the exciting electric field. ${ }^{5}$

The present letter studies the question whether a similar approach, namely coating a fluorescent bead with a metal layer, can enhance the photostability of an embedded dye without sacrificing too much of the detectable fluorescence by energy absorption within the metal. For the sake of simplicity, a single dye molecule centered within a dielectric bead coated with a thin metal layer is considered; see Fig. 1. The molecule's fluorescence excitation and emission is treated as that of a classical electric dipole, with collinear absorption and emission dipole axis. Fluorescence is excited by a plane electromagnetic wave incident onto the bead with its electric field amplitude vector parallel to the molecule's dipole axis.

When calculating the fluorescence properties of the metal/bead/dye system, three effects must be taken into account: (i) the changed intensity of the exciting electric field at the molecule's position due to the interaction of the incident electromagnetic wave with the metal coating; (ii) the altered lifetime of the excited state of the molecule due to its electromagnetic interaction with the metal; (iii) the partial absorption of emitted energy within the metal coating. The

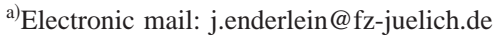

electric field inside the bead is calculated in a standard way ${ }^{6}$ by representing the incident plane wave and the induced electromagnetic fields within the different, dielectrically homogeneous regions (free space, metal coating, bead interior) as series of vector spherical harmonics, while requiring matching of the boundary conditions (continuity of tangential values of the electric and magnetic fields at all interfaces). Thus, Maxwell's equations are solved exactly, taking into account also retardation effects. Then, the excitation rate of the molecule is given by the square of the scalar product of the electric field amplitude $E_{\mathrm{ex}}$ (at the molecule's position) and the molecule's absorption dipole moment. Here, sufficiently low excitation intensity is assumed so that optical saturation of the molecule can be neglected.

Treatment of the fluorescence emission is done within the framework of classical electrodynamics, which has proven to be extremely successful for similar problems in the past; see, e.g., Refs. 7 and 8. The computational procedure can be described as follows. First, the electromagnetic emission of the molecular dipole is calculated by solving Maxwell's equations within the given geometry; see, e.g., Ref. 9. By determining the energy flux through the inner dielectric/ metal boundary (integrating the radial part of the Poynting vector over the boundary), one obtains the complete energy flux through this surface. The lifetime of the excited state is inversely proportional to that energy flux. If the bead without metal coating is taken as a reference, one has the relation $\tau / \tau_{0}=S_{\mathrm{in}, 0} / S_{\mathrm{in}}$, where $\tau, \tau_{0}$ and $S_{\mathrm{in}}, S_{\mathrm{in}, 0}$ are the lifetime
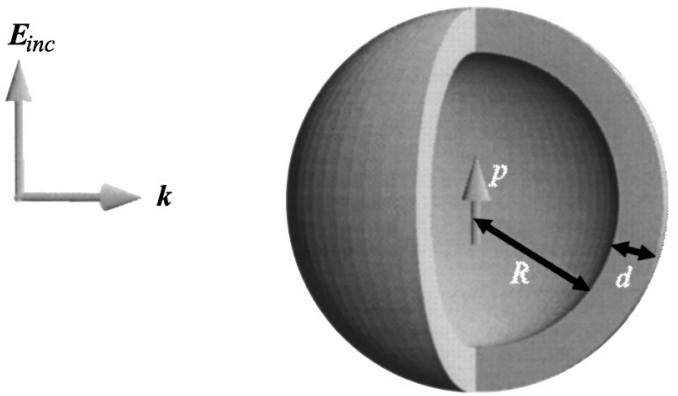

FIG. 1. Picture of the problem considered: A plane electromagnetic wave is incident onto a spherical dielectric bead with a metal coating. At the bead's center, a dye molecule is positioned, with its excitation/emission dipole parallel to the electric field vector of the plane wave. 


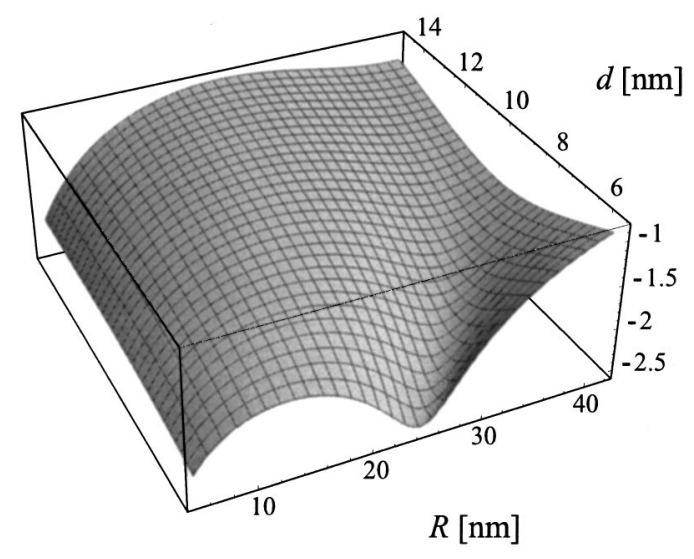

FIG. 2. Dependence of the decadic logarithm of lifetime ratio $\tau / \tau_{0}$ upon bead radius $R$ and metal layer thickness $d$.

and energy flux values for the coated and uncoated bead, respectively. For the sake of simplicity, it was assumed that all the energy of the excited state is converted into electromagnetic emission (corresponding to a fluorescence quantum yield of one). Second, the energy flux $S_{\text {out }}$ through the outer metal boundary is calculated, again by integrating the radial part of the Poynting vector over that surface. The ratio $\Phi_{f}$ $=S_{\text {out }} / S_{\text {in }}$ gives the part of the emitted energy that is not absorbed within the metal. Note that the value of $\Phi_{f}$ is always one for the uncoated bead-the dielectric material of the bead is assumed to be optically nonabsorptive. Third, the detectable fluorescence intensity $I$ is proportional to the product of $\Phi_{f}$ and $E_{\mathrm{ex}}^{2}$, thus yielding

$$
\frac{I}{I_{0}}=\Phi_{f} \frac{E_{\mathrm{ex}}^{2}}{E_{\mathrm{ex}, 0}^{2}}=\frac{S_{\mathrm{out}} E_{\mathrm{ex}}^{2}}{S_{\mathrm{in}} E_{\mathrm{ex}, 0}^{2}},
$$

where, again, the subscript naught relates to the uncoated bead. An important quantity is the average number $N$ of detectable fluorescence photons emitted by the molecule until photobleaching. If one assumes, as stated in the introduction, that photobleaching occurs only while the molecule is in its excited state, this number is inversely proportional to the excited state lifetime, multiplied by $\Phi_{f}$ (fraction of emitted photons not absorbed by the metal layer). Thus, one obtains

$$
\frac{N}{N_{0}}=\Phi_{f} \frac{\tau_{0}}{\tau}=\frac{S_{\text {out }}}{S_{\text {in, }, 0}}
$$

where $N_{0}$ is the average number for the uncoated bead.

Numerical calculations were performed for the following model parameters: excitation wavelength $635 \mathrm{~nm}$ (typical diode laser wavelength), emission wavelength $670 \mathrm{~nm}$ (taking into account a Stokes shift of the fluorescence emission with respect to the excitation wavelength). The bead consists of a dielectric medium (e.g., polymer) with index of refraction $n_{i}=1.5$. The metal coating is assumed to be silver, with complex valued index of refraction $n_{m}=0.157+3.82 i$ at $635 \mathrm{~nm}$ and $n_{m}=0.163+4.07 i$ at $670 \mathrm{~nm} .{ }^{10}$ The metal-coated bead is assumed to be suspended in water with index of refraction $n_{w}=1.33$. In Fig. 2, the lifetime ratios $\tau / \tau_{0}$ are plotted for varying values of bead radius and metal layer thickness. As can be seen, a dramatic decrease in excited state lifetime occurs for specific values of bead radius and layer thickness.

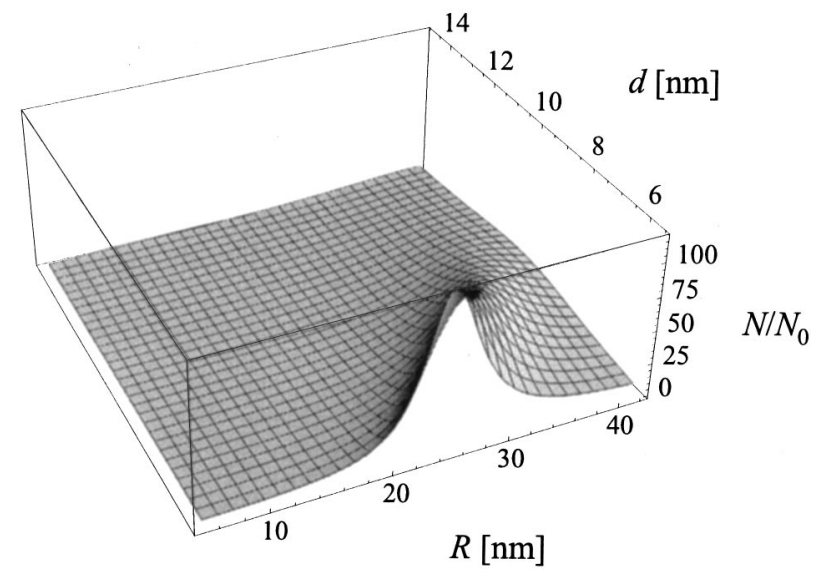

FIG. 3. Dependence of the ratio $N / N_{0}$ of average emitted fluorescence photons until photobleaching upon bead radius $R$ and metal layer thickness $d$.

Figure 3 shows the calculated ratio $N / N_{0}$ of the average numbers of emitted photons until photobleaching. Despite a fluorescence yield smaller than one, the shortening in lifetime is much larger than the fluorescence losses caused by energy absorption within the metal, thus yielding a dramatic increase of the average number of fluorescence photons that the molecule can emit until photobleaching. Finally, the calculated ratio of fluorescence intensities, $I / I_{0}$, is presented in Fig. 4. At favorite values of bead diameter and shell thickness, the coated bead yields roughly a 20 times higher fluorescence intensity than the uncoated bead, despite the absorption losses within the metal. This is due to the significant enhancement of the electric field amplitude at the bead's center.

It should be noted that the bead size considered in the present letter is much smaller than the excitation/emission wavelength, thus being far away from values where Mie resonances come into play. ${ }^{11}$ The fluorescence enhancement considered here is a dominantly near-field effect, very similar to that assumed to be important in surface enhanced Raman scattering $^{12,13}$ and observed in near-field enhanced fluorescence. ${ }^{14-16}$ The refractive index of the metal coating was assumed to be equal to the bulk values of silver, and the calculations were done in a continuous medium approximation. However, this should yield reliable results for layer

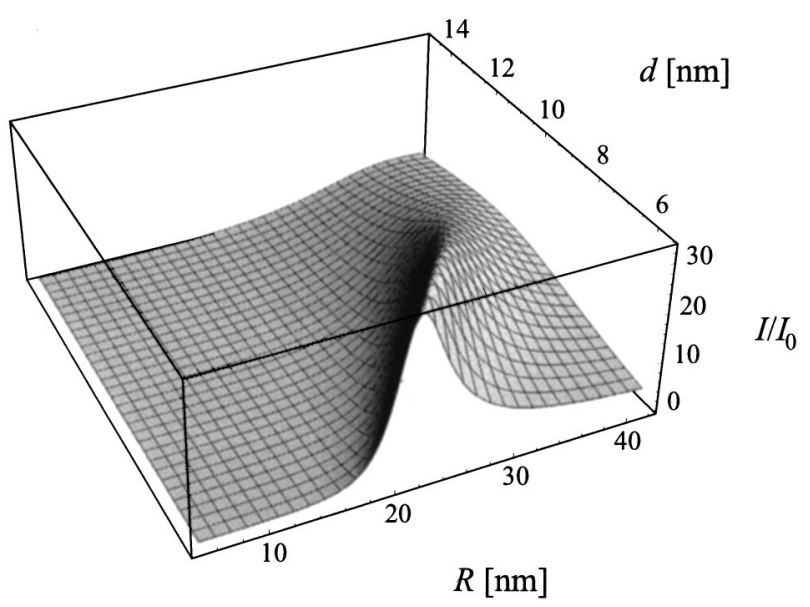

FIG. 4. Dependence of intensity ratio $I / I_{0}$ upon bead radius $R$ and metal layer thickness $d$.

o AlP license or copyright, see http://apl.aip.org/apl/copyright.jsp 
thickness values down to $5 \mathrm{~nm} \cdot{ }^{17,18}$ In the present work, only the simplified case of a dye molecule located directly at the center of the embedding bead was considered. However, in many real life applications, fluorescent molecules are homogeneously distributed over the whole bead volume. Treating this more general situation is computationally much more demanding than the case studied here. Extensive numerical results for this more general case will be presented elsewhere.

The results presented can be important for the design of a new class of fluorescent labels with significantly higher photostability and increased fluorescence intensity, and for improving the optical properties of fluorescing semiconductor nanocrystals. ${ }^{19}$ For example, the dramatic decrease of the excited state lifetime can significantly lower the probability of switching into nonfluorescent dark states, an issue of considerable concern for fluorescent nanocrystals. ${ }^{20}$ Furthermore, the enhancement of the fluorescence properties of nanoparticles may prove useful within the context of achieving stimulated emission in fluorescent nanoparticle aggregates. $^{21}$

Financial support by the Volkswagenstiftung (Grant No. $\mathrm{I} / 76064)$ is gratefully acknowledged. The author thanks Martin Böhmer for many inspiring discussions and would like to thank Eike Stedefeldt for his enduring and exceedingly helpful support of the work.

${ }^{1}$ E. M. Purcell, Phys. Rev. 69, 681 (1947).

${ }^{2}$ K. H. Drexhage, Prog. Opt. XII, 165 (1974).

${ }^{3}$ D. Kleppner, Phys. Rev. Lett. 47, 233 (1981).

${ }^{4}$ J. Enderlein, Chem. Phys. 247, 1 (1999).

${ }^{5}$ J. Enderlein, Biophys. J. 78, 2151 (2000).
${ }^{6}$ H. C. van de Hulst, Light Scattering by Small Particles (Dover, New York, 1981).

${ }^{7}$ R. R. Chance, A. Prock, and R. Silbey, Adv. Chem. Phys. 37, 1 (1978).

${ }^{8}$ S. D. Brorson, in Spontaneous Emission and Laser Oscillation in Microcavities, edited by H. Yokoyama and K. Ujihara (CRC Press, Boca Raton, FL, 1995), p. 151.

${ }^{9}$ K. G. Sullivan and D. G. Hall, J. Opt. Soc. Am. B 14, 1149 (1997); 14, 1160 (1997).

${ }^{10}$ A. D. Rakic, A. B. Djurišic, J. M. Elazar, and M. L. Majewski, Appl. Opt. 37, 5271 (1998).

${ }^{11}$ A. J. Campillo, J. D. Eversole, and H. B. Lin, Phys. Rev. Lett. 67, 437 (1991); H. B. Lin, J. D. Eversole, C. D. Merritt, and A. J. Campillo, Phys. Rev. A 45, 6756 (1992).

${ }^{12}$ M. Moskovits, Rev. Mod. Phys. 57, 783 (1985).

${ }^{13}$ K. Kneipp, Y. Wang, H. Kneipp, L. T. Perelman, I. Itzkan, R. R. Dasari, and M. S. Feld, Phys. Rev. Lett. 78, 1667 (1997); K. Kneipp, H. Kneipp, R. Manoharan, E. B. Hanlon, I. Itzkan, R. R. Dasari, and M. S. Feld, Appl. Spectrosc. 52, 1493 (1998).

${ }^{14}$ J. Azoulay, A. Debarre, and P. Tchenio, J. Microsc. 194, 486 (1999).

${ }^{15}$ H. F. Hamann, A. Gallagher, and D. J. Nesbitt, Appl. Phys. Lett. 76, 1953 (2000); H. F. Hamann, M. Kuno, A. Gallagher, and D. J. Nesbitt, J. Chem. Phys. 114, 8596 (2001).

${ }^{16}$ E. J. Sanchez, L. Novotny, and X. S. Xie, Phys. Rev. Lett. 82, 4014 (1999).

${ }^{17}$ C. Girard, A. Dereux, and O. J. F. Martin, Phys. Rev. B 49, 13872 (1994).

${ }^{18}$ F. J. Garcia-Vidal and J. B. Pendry, Phys. Rev. Lett. 77, 1163 (1996).

${ }^{19}$ M. G. Bawendi, W. L. Wilson, L. Rothberg, P. J. Carroll, T. M. Jedju, M. L. Steigerwald, and L. E. Brus, Phys. Rev. Lett. 65, 1623 (1990); S. A. Empedocles, D. J. Norris, and M. G. Bawendi, ibid. 77, 3873 (1996); J. Rodriguez-Viejo, K. F. Jensen, H. Mattoussi, J. Michel, B. O. Dabbousi, and M. G. Bawendi, Appl. Phys. Lett. 70, 2132 (1997).

${ }^{20}$ M. Nirmal, B. O. Dabbousi, M. G. Bawendi, J. J. Macklin, J. K. Trautman, T. D. Harris, and L. E. Brus, Nature (London) 383, 802 (1996); M. Kuno, D. P. Fromm, H. F. Hamann, A. Gallagher, and D. J. Nesbitt, J. Chem. Phys. 112, 3117 (2000).

${ }^{21}$ V. I. Klimov, A. A. Mikhailovsky, S. Xu, A. Malko, J. A. Hollingsworth, C. A. Leatherdale, H. J. Eisler, and M. G. Bawendi, Science 290, 314 (2000). 